\title{
Acetate transiently inhibits myocardial contraction by increasing mitochondrial calcium uptake
}

\author{
James F Schooley ${ }^{1}$, Aryan M A Namboodiri ${ }^{1}$, Rachel T Cox ${ }^{2}$, Rolf Bünger ${ }^{1}$ and Thomas P Flagg ${ }^{1 *}$
}

\begin{abstract}
Background: There is a close relationship between cardiovascular disease and cardiac energy metabolism, and we have previously demonstrated that palmitate inhibits myocyte contraction by increasing $K_{v}$ channel activity and decreasing the action potential duration. Glucose and long chain fatty acids are the major fuel sources supporting cardiac function; however, cardiac myocytes can utilize a variety of substrates for energy generation, and previous studies demonstrate the acetate is rapidly taken up and oxidized by the heart. In this study, we tested the effects of acetate on contractile function of isolated mouse ventricular myocytes.

Results: Acute exposure of myocytes to $10 \mathrm{mM}$ sodium acetate caused a marked, but transient, decrease in systolic sarcomere shortening (1.49 $\pm 0.20 \%$ vs. $5.58 \pm 0.49 \%$ in control), accompanied by a significant increase in diastolic sarcomere length $(1.81 \pm 0.01 \mu \mathrm{m}$ vs. $1.77 \pm 0.01 \mu \mathrm{m}$ in control), with a near linear dose response in the 1-10 $\mathrm{mM}$ range. Unlike palmitate, acetate caused no change in action potential duration; however, acetate markedly increased mitochondrial $\mathrm{Ca}^{2+}$ uptake. Moreover, pretreatment of cells with the mitochondrial $\mathrm{Ca}^{2+}$ uptake blocker,

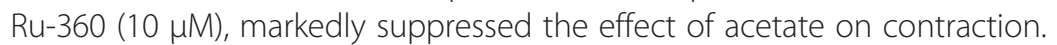

Conclusions: Lehninger and others have previously demonstrated that the anions of weak aliphatic acids such as acetate stimulate $\mathrm{Ca}^{2+}$ uptake in isolated mitochondria. Here we show that this effect of acetate appears to extend to isolated cardiac myocytes where it transiently modulates cell contraction.
\end{abstract}

\section{Background}

It is well established that the cardiac myocardium is capable of oxidizing a variety of carbon sources to supply the energy required for continuous contraction. Lipids, carbohydrates, ketone bodies, and amino acids can all support some degree of ATP synthesis in the heart. The loss of metabolic flexibility in the diseased heart may lead to abnormal contractile function. For example, we recently demonstrated that mice overexpressing fatty acid transport protein (FATP4) in the heart have impaired diastolic function [1]. Similarly, acute exposure to long chain fatty acids has been shown to cause a decrease in cardiomyocyte contractility through effects on increases in voltage gated $\mathrm{K}^{+}$currents thus causing shortening of the action potential [2].

\footnotetext{
* Correspondence: Thomas.Flagg@usuhs.edu

'Department of Anatomy, Physiology, and Genetics, Uniformed Services University for the Health Sciences, 4301 Jones Bridge Road, Rm. C-2114, Bethesda 20814, MD, USA

Full list of author information is available at the end of the article
}

In a continued effort to understand the relationship of cardiac metabolism and cell function, we set out to test the effects of acetate on contraction. Several studies have examined the effect of acetate on cardiac contraction with equivocal results. In isolated cells, acetate tends to increase cell shortening after 10 minutes of exposure [3]. In isolated papillary muscle and in vivo, sodium acetate has been shown to reduce contractility $[4,5]$, whereas other studies suggest that acetate causes an increase in contractility [6]. The effects of acetate on cardiovascular function in vivo are complicated by the concomitant vasodilatory effects that also must be considered [6]. These mixed results are consistent with the idea that acetate can affect cardiac contractility, but the cellular mechanisms remain poorly understood.

Although it is typically found in low concentrations $(\sim 0.2 \mathrm{mM})$ in non-ruminant mammals [7], acetate oxidation can account for $\sim 10 \%$ of the total $\mathrm{CO}_{2}$ output in humans [8]. Acetate can be converted to acetyl CoA by acetyl CoA sythetase (AceCS2) in the mitochondrial matrix [9] and the resultant acetyl CoA can then enter 
the tricarboxylic acid (TCA) cycle. The heart is unique in that the expression of the mitochondrial AceCS2 is higher than in any other tissue, so the heart is ideally suited to use acetate as a fuel source [9]. Metabolic studies by Randle demonstrate that acetate is rapidly oxidized in the myocardium [10]. In some isolated heart studies, acetate combustion can account for $\sim 90 \%$ of total respiration to the exclusion of glucose oxidation [11], although others suggest that at physiological workloads both acetate and glucose are effectively utilized [12]. Acetate can also affect isolated mitochondria independent of its oxidation, where Lehninger and others have demonstrated that exposure to acetate causes a rapid increase in mitochondrial matrix $\mathrm{Ca}^{2+}$ and osmotic swelling [13-15].

In this context, we investigated the effects of acetate on cardiac contractility in isolated cardiac myocytes. We tested the effects of acetate throughout a 10 minute exposure. The results demonstrate that acetate inhibits systolic function and increases cell relaxation within $2 \mathrm{mi}-$ nutes of exposure. The decrease in systolic function is transient, however, and contraction amplitude is restored within 10 minutes. These effects are independent of changes in action potential duration; however, the effects of acetate were inhibited by blockade of mitochondrial $\mathrm{Ca}^{2+}$ uptake with $\mathrm{Ru}-360$, indicating that acetate causes effects on cardiac contraction by increasing $\mathrm{Ca}^{2+}$ uptake into the mitochondria.

\section{Methods}

\section{Animal subjects}

All animals used in this study were male, aged 2-4 months, C57Bl6/J. All procedures complied with the standards for the care and use of animal subjects as stated in the Guide for the Care and Use of Laboratory Animals (NIH publication No. 85-23, revised 1996). Protocols were approved by the USUHS Institutional Animal Care and Use Committee.

\section{Solutions (concentrations in $\mathrm{mM}$ )}

Normal Tyrode Soution (NT): $\mathrm{NaCl}, 137 ; \mathrm{KCl}, 5.4 ; \mathrm{NaH}_{2} \mathrm{PO}_{4}$, 0.16; glucose, $10 ; \mathrm{MgCl}_{2}, 0.5 ; \mathrm{CaCl}_{2}, 1.8$; HEPES, 5.0; $\mathrm{NaHCO}_{3}, 3.0 ; \mathrm{pH} 7.35$ - 7.4.

Wittenberg Isolation Medium (WIM): $\mathrm{NaCl}, 116 ; \mathrm{KCl}$, 5.3; $\mathrm{NaH}_{2} \mathrm{PO}_{4}, 1.2$; glucose, 11.6; $\mathrm{MgCl}_{2}$, 3.7; HEPES, 20; L-glutamine, 2.0; $\mathrm{NaHCO}_{3}, 4.4 ; \mathrm{KH}_{2} \mathrm{PO}_{4}, 1.5 ; 1 \mathrm{X}$ essential vitamins; $1 \mathrm{X}$ amino acids; $\mathrm{pH}$ 7.3-7.4.

\section{Myocyte isolation}

Isolation of ventricular myocytes was performed as described previously $[1,2,16]$. Briefly, mice were anesthetized by intra-peritoneal injection with 2,2,2 tribromoethanol $(250 \mathrm{mg} / \mathrm{kg})$. Following cervical dislocation, the heart was rapidly excised and the aorta cannulated. The heart was retrogradely perfused with $\mathrm{Ca}^{2+}$-free WIM solution for
5 minutes followed by perfusion with a digestion solution containing $100 \mu \mathrm{M} \mathrm{CaCl}_{2}$ and $1 \mathrm{mg} / \mathrm{mL}$ collagenase (Type 2, Worthington Biochemical). Left ventricular cells were gently dispersed by manual trituration using a pasteur pipette in WIM solution supplemented with bovine serum albumin $(1 \mathrm{mg} / \mathrm{mL})$ and $500 \mu \mathrm{M} \mathrm{CaCl}$. Cells were washed twice with WIM solution and twice with HEPES-buffered M199 solution and stored at room temperature. Cells were used for experiments within 12 hours of isolation in all cases.

\section{Myocyte contraction measurements}

Unloaded sarcomere shortening was measured in freshly isolated ventricular myocytes, as described previously $[1,2]$. Briefly, isolated myocytes were transferred into a recording chamber mounted on an Olympus X51 inverted microscope and superfused with normal Tyrode solution saturated with room air. Additions to the Tyrode solution are described in the text. The mitochondrial calcium uptake inhibitor, $\mathrm{Ru}-360$, was obtained from EMD Biosciences; all other chemicals were purchased from Sigma. Typically, cells were field stimulated to contract at $1 \mathrm{~Hz}$. When thapsigargin was applied to cells, the stimulation frequency was reduced to $0.5 \mathrm{~Hz}$. Video images were acquired using a Myocam camera and IonWizard software (IonOptix, Inc.). All experiments were performed at room temperature.

\section{Mitochondrial $\mathrm{Ca}^{2+}$ measurements}

Freshly isolated ventricular myocytes were plated on laminin-coated $(100 \mu \mathrm{g} / \mathrm{mL})$ Mat-Tek dishes for fluorescence imaging. Cells were loaded with Rhod-2-AM ( $5 \mu \mathrm{M})$ for 30 minutes in normal Tyrode solution containing probenecid $(500 \mu \mathrm{M})$ to inhibit dye export and $200 \mu \mathrm{M} \mathrm{MnCl}_{2}$ to quench cytoplasmic fluorescence as has been previously reported [17,18]. After loading, cells were washed twice with normal Tyrode solution supplemented with probenecid and $200 \mu \mathrm{M} \mathrm{MnCl}_{2}$ and transferred to the microscope stage. Cell images were obtained every 10 seconds for 10 minutes. Data were plotted as background-subtracted Rhod-2-AM fluorescence normalized to mean signal during the first 6 images recorded prior to addition of acetate.

\section{Action potential measurements}

Action potentials were measured in freshly isolated ventricular myocytes using whole cell current clamp. Briefly, following acquisition of the whole cell mode, cell holding potential was adjusted to $-70 \mathrm{mV}$ using current injection. Action potentials were evoked by suprathreshold stimuli $(2 \mathrm{nA}, 3 \mathrm{msec})$ delivered at $1 \mathrm{~Hz}$. Action potentials were recorded continuously during 5 minute exposure to acetate followed by 5 minutes washout. Average traces constructed from 25 consecutive action potentials during control, acetate exposure ( 2 minutes) and washout ( 5 minutes) were 
analyzed. Action potential duration $\left(\mathrm{APD}_{90}\right)$ was determined at $90 \%$ repolarization and referenced to the peak of the action potential.

\section{Data analysis}

All data were analyzed using ClampFit, IonWizard, Image and Microsoft Excel software and (except where noted) results are presented as mean \pm SEM (standard error of the mean). Statistical analysis was performed with built-in functions of Excel or with the Sigma XL software add-in. Statistical tests and p-values are denoted in the figure legend and text where appropriate.

\section{Results}

Acute exposure to acetate transiently impairs cardiac contraction and increases diastolic sarcomere length

To test whether the short chain fatty acid, acetate, exerts negative inotropic effects, we continuously monitored average sarcomere length in isolated mouse cardiomyocytes acutely exposed to Tyrode solution containing $10 \mathrm{mM}$ sodium acetate. Three major consequences of acetate exposure were observed. Figure 1 shows that acetate caused a transient decrease in active sarcomere shortening. At two minutes following acetate application, fractional shortening was markedly decreased from $5.6 \pm 0.5$ to $1.5 \pm 0.2(n=12$, $\mathrm{p}<0.001$, paired t-test). In the continued presence of acetate, contraction amplitude gradually recovered and returned to baseline after approximately 10 minutes. We also noted a marked increase in fractional sarcomere shortening when acetate was removed from the bath solution.
In addition, exposure to acetate significantly increased the diastolic sarcomere length. We next examined the concentration dependence of the decrease in contraction observed at two minutes and diastolic sarcomere length following the exposure to acetate. Figure 2 shows that the negative inotropic effect of acetate is concentration dependent. Data were fit with a modified Hill equation with an $\mathrm{IC}_{50}=5.6 \mathrm{mM}$ and Hill coefficient of 1.4. There was no apparent concentration dependence for the effect on diastolic sarcomere length.

\section{Acetate exposure does not affect the action potential duration}

We previously demonstrated that acute exposure to long chain fatty acids shortens the action potential duration principally by increasing outward voltage-dependent $\mathrm{K}^{+}$ currents encoded by $K_{\mathrm{v}} 2.1$ and $\mathrm{K}_{\mathrm{v}} 1.5$, with no effect on $\mathrm{IK}_{1}$ [2]. Considering that short-chain fatty acids might have similar effects on cell excitability, we examined the effect of acetate on the action potential duration $\left(\mathrm{APD}_{90}\right)$ (Figure 3$)$. In contrast to results with palmitate, $\mathrm{APD}_{90}(24.5 \pm 3.2 \mathrm{msec}$ in control) was unaffected by acetate $(25.0 \pm 3.5 \mathrm{msec}, \mathrm{p}>0.05$, paired t-test), suggesting that a different molecular mechanism underlies the inotropic effects of acetate.

\section{Acetate exposure stimulates mitochondrial $\mathrm{Ca}^{2+}$ uptake}

It has been shown previously that acetate increases $\mathrm{Ca}^{2+}$ uptake in isolated liver and heart mitochondria [13-15]. In this light, we hypothesized that the acute application
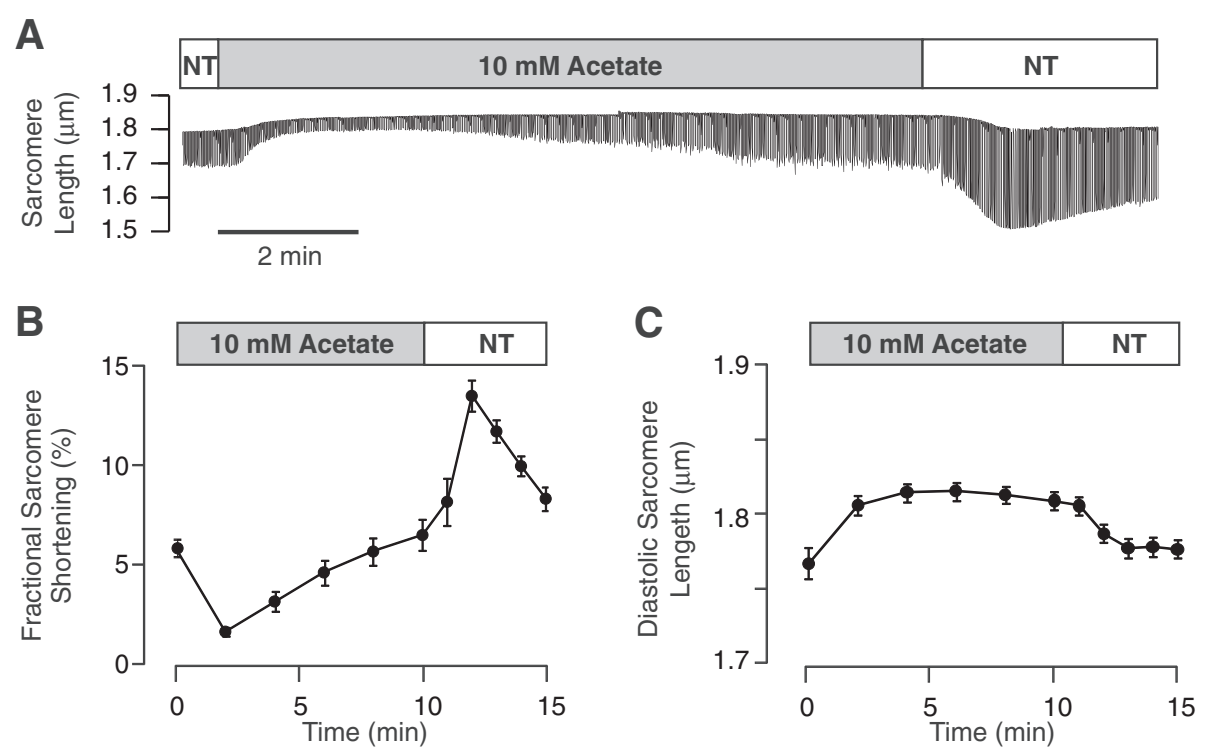

Figure 1 Acetate causes a transient decrease of fractional sarcomere shortening and an increase in diastolic sarcomere length in isolated mouse cardiac myocytes. (A) Representative recording of average sarcomere length assessed continuously throughout application and removal of normal Tyrode solution (NT) supplemented with $10 \mathrm{mM}$ sodium acetate. Summary data from experiments as in $\mathbf{A}(n=15)$, illustrating the effects of acetate on (B) fractional sarcomere shortening and (C) diastolic sarcomere length. 

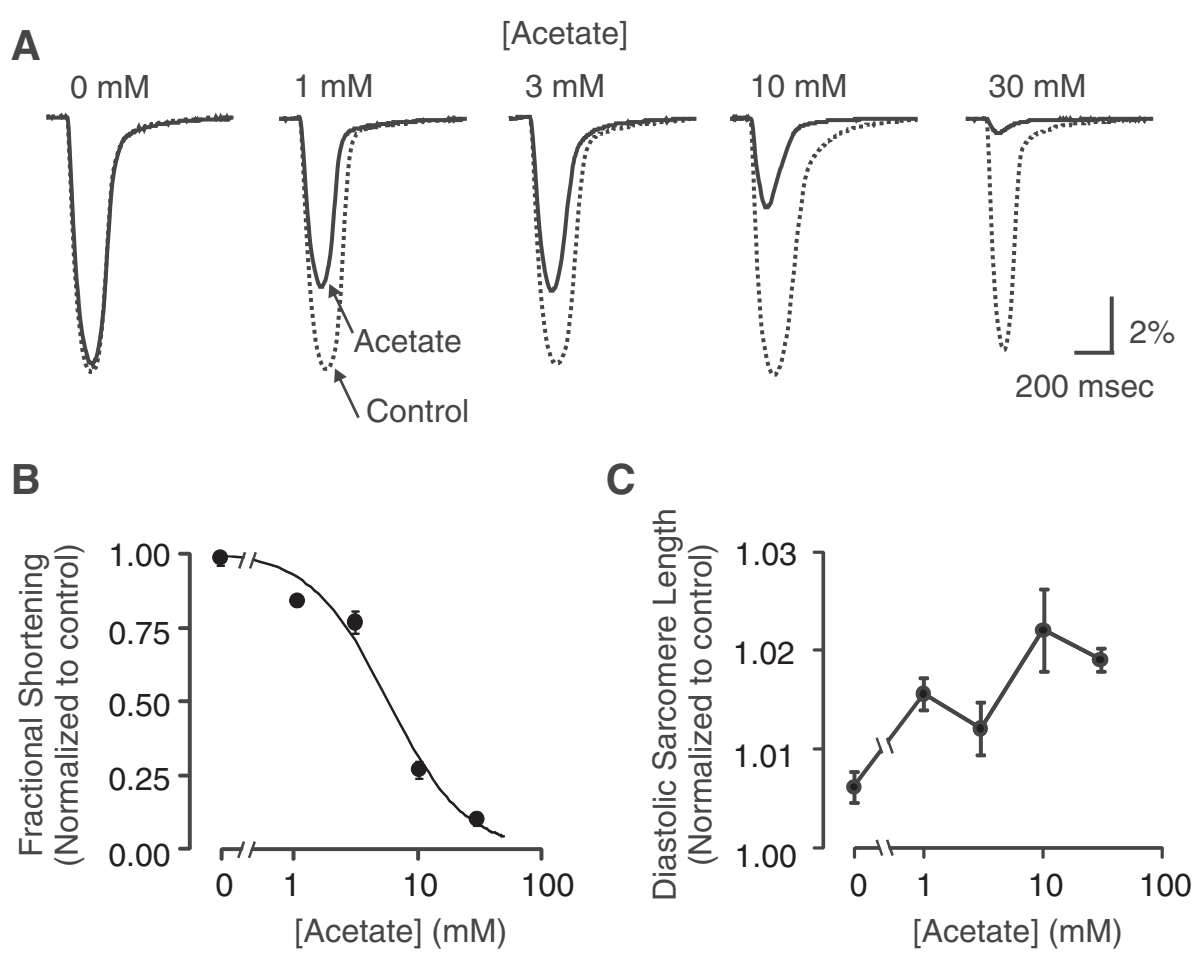

Figure 2 Concentration-dependence of acetate effect on fractional shortening and diastolic sarcomere length. (A) Single averaged contractions acquired in experiments as described in Figure 1 at different concentrations of sodium acetate. Contractions in normal Tyrode (Control, dotted line) and at 2 minutes following exposure to acetate solution (Acetate, solid line) are shown. $10 \mathrm{mM} \mathrm{NaCl}$, instead of sodium acetate, was added to normal Tyrode to collect the zero acetate data. (B) Acetate concentration response curve for maximum contraction inhibition. Data were fit with a modified Hill equation (solid line): $F S / F S_{0}=1 /\left(1+\left([\text { Acetate }] / I C_{50}\right)^{h}\right)$, where $I C_{50}$ is the half-maximal inhibitory concentration of acetate $\left(I_{50}=5.6 \mathrm{mM}\right)$ and $\mathrm{h}$ is the Hill coefficient $(\mathrm{h}=1.3)$. (C) There was no apparent acetate concentration dependence on diastolic sarcomere length.
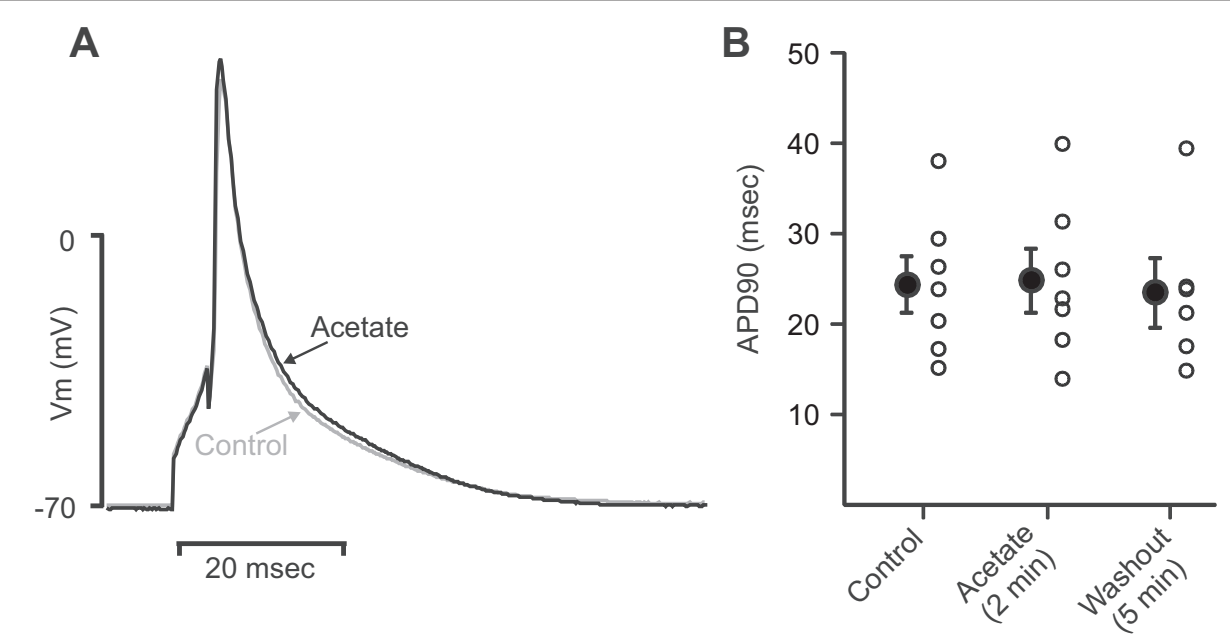

Figure 3 Acetate does not affect the action potential duration ( $\left(\mathbf{P P D}_{\mathbf{9 0}}\right.$ ). (A) Representative action potentials recorded before (Control, gray line) and 2 minutes following acetate exposure (Acetate, black line). Action potentials were recorded in whole cell current clamp mode and were evoked suprathreshold current injections. Records are averaged traces from 25 consecutive action potentials. (B) Summary APD 90 data from all $(n=7)$ experiments as in $\mathbf{A}$. Acetate exposure did not affect the $\mathrm{APD}_{90}(24.5 \pm 3.2 \mathrm{msec}$ in acetate vs. $25.0 \pm 3.5 \mathrm{msec}$ in control). 
of acetate might lead to an increase in mitochondrial $\mathrm{Ca}^{2+}$ uptake, leaving less $\mathrm{Ca}^{2+}$ available to activate the myofilaments. To test this hypothesis, we first monitored mitochondrial $\mathrm{Ca}^{2+}$ during exposure to acetate using the fluorescent $\mathrm{Ca}^{2+}$ indicator, Rhod-2 AM $(5 \mu \mathrm{M})$ in the presence of $200 \mu \mathrm{M} \mathrm{MnCl}$ to quench cytosolic fluorescence $[17,18]$. Figure 4 shows normalized cell fluorescence during exposure to $10 \mathrm{mM}$ acetate in the presence or absence of the mitochondrial $\mathrm{Ca}^{2+}$ uptake inhibitor, Ru-360 ( $\mathrm{n}=9$ and 8 respectively). This concentration of $\mathrm{Ru}-360$ was chosen as it has previously been shown to have no effect on transmembrane $\mathrm{Ca}^{2+}$ fluxes other than mitochondrial uptake $[19,20]$. The results indicate that acetate causes a specific increase in Rhod-2 fluorescence consistent with the conclusion that acetate increases mitochondrial $\mathrm{Ca}^{2+}$ uptake.

\section{Inhibition of mitochondrial $\mathrm{Ca}^{2+}$ uptake attenuates the effects of acetate on fractional shortening and diastolic sarcomere length}

If the acetate-dependent increase in mitochondrial $\mathrm{Ca}^{2+}$ is responsible for the reduction of myocyte fractional shortening, we reasoned that inhibiting mitochondrial $\mathrm{Ca}^{2+}$ uptake would reduce or abolish the effect of acetate on myocyte contraction. To test this hypothesis, we incubated cells for 30 minutes with $10 \mu \mathrm{M} \mathrm{Ru}-360$, and then measured the effects of acetate on sarcomere shortening. Figure 5 shows that Ru-360 treatment significantly attenuated the effect of acetate on both diastolic sarcomere length and fractional sarcomere shortening. Interestingly, pretreatment with Ru-360 alone also had a marked effect on contractile function prior to acetate exposure. As shown in Figure 5B (inset), fractional sarcomere shortening immediately prior to acetate exposure (time zero) was
$13.03 \pm 1.17 \%$ in cells pretreated with $\mathrm{Ru}-360$ compared with $5.83 \pm 0.43 \%$ in control cells not exposed to $\mathrm{Ru}-360$. Taken together, these data support the conclusion that acetate stimulates mitochondrial $\mathrm{Ca}^{2+}$ uptake, leading to reduced availability of $\mathrm{Ca}^{2+}$ for myofilament activation.

\section{Partial inhibition of SERCA inhibits recovery of systolic function during acetate application}

The acetate-induced stimulation of mitochondrial $\mathrm{Ca}^{2+}$ uptake appears to be the seminal event leading to reduced inotropy. However, in the sustained presence of acetate, fractional shortening recovers to control levels by 10 minutes. This suggests that during the early phase of acetate exposure, cytosolic $\mathrm{Ca}^{2+}$ released from the SR bypasses the myofilaments and enters the mitochondria. We hypothesized that the recovery of contraction during the latter phase of acetate exposure reflects refilling of the SR to replace the $\mathrm{Ca}^{2+}$ lost to the mitochondria. Previous studies have utilized brief application of $1 \mu \mathrm{M}$ thapsigargin to partially inhibit SERCA activity [21]. We therefore treated cells with $1 \mu \mathrm{M}$ thapsigargin for $2-5$ minutes prior to transfer to the bath for recording. No thapsigargin was added to the recording solutions. As expected, cell relaxation was markedly slowed by SERCA inhibition and therefore cells were stimulated at $0.5 \mathrm{~Hz}$ (instead of $1 \mathrm{~Hz}$ ) for these experiments (Figure 6). Thapsigargin pretreatment markedly inhibited fractional shortening as expected (Figure 6B, inset), but exposure to acetate still resulted in a decrease in contraction amplitude and increase in diastolic sarcomere length similar to control, untreated cells. It should be noted, however, that the recovery of contraction typically observed during the latter phase of acetate application as well as the transient increase in contraction at washout were

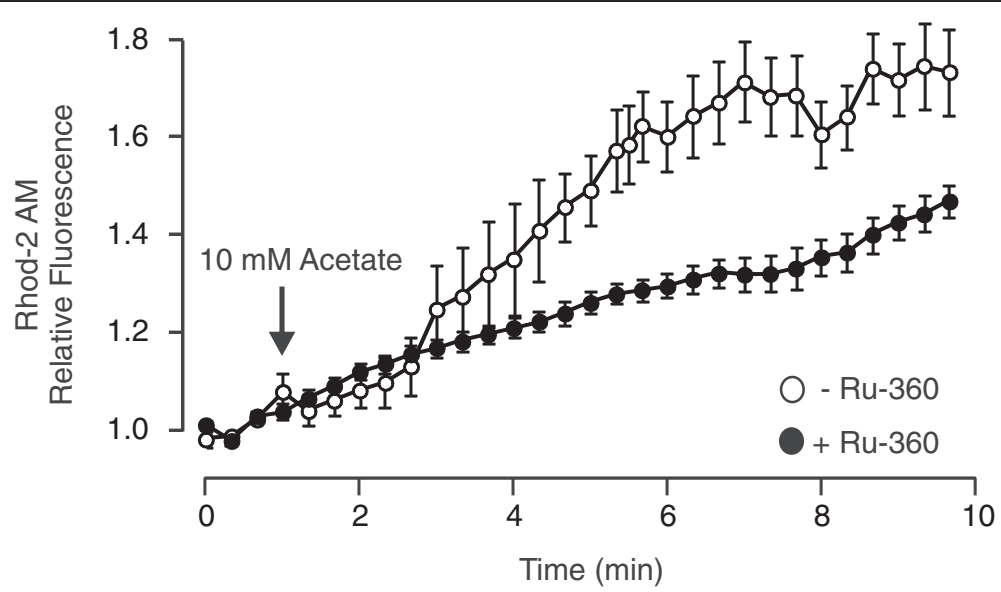

Figure 4 Acetate stimulates mitochondrial $\mathrm{Ca}^{2+}$ uptake. Summary of rhod-2-AM fluorescence recorded during exposure to 10 mM sodium acetate in the presence $(n=8)$ or absence $(n=10)$ of Ru-360 $(10 \mu M)$. Background-subtracted fluorescence was normalized to mean fluorescence during the period prior to addition of $10 \mathrm{mM}$ acetate $(n=10)$ or control (no acetate, $n=9)$. Acetate solution was added at the arrow. 


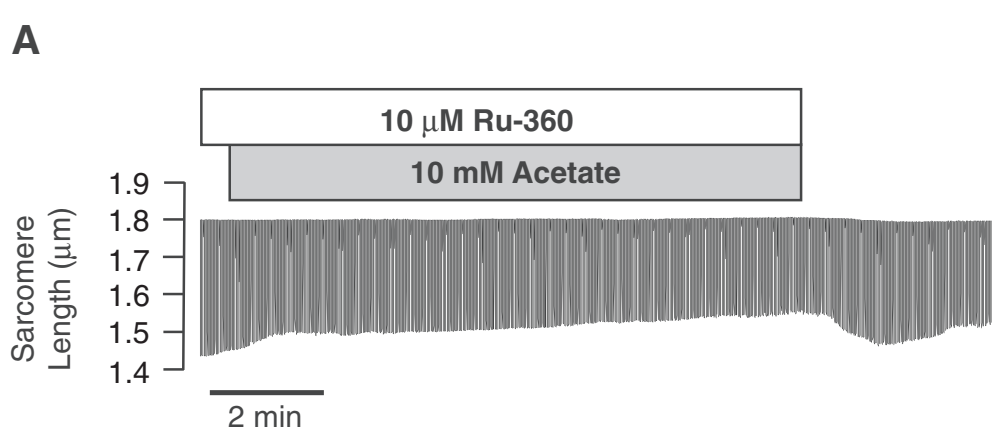

B

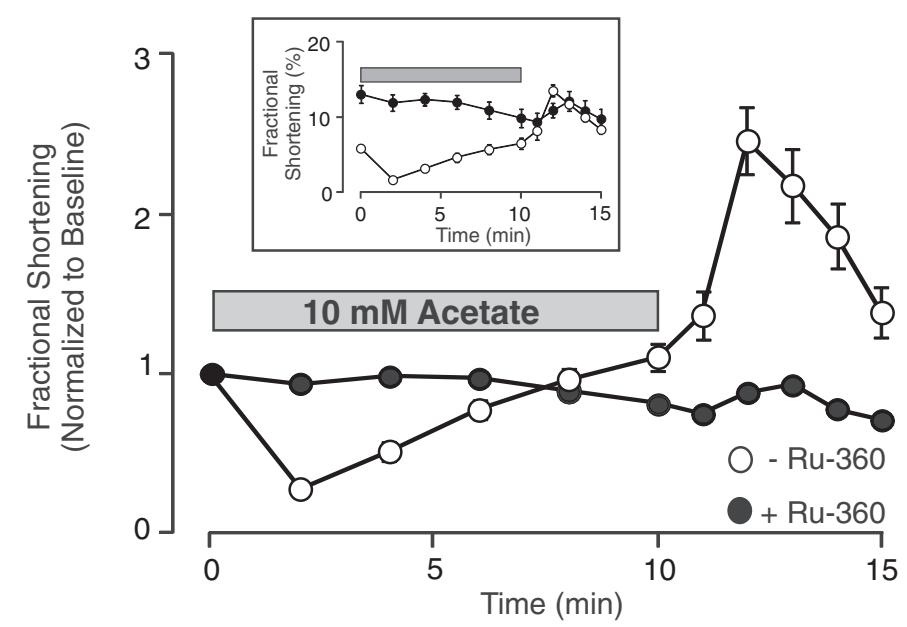

C

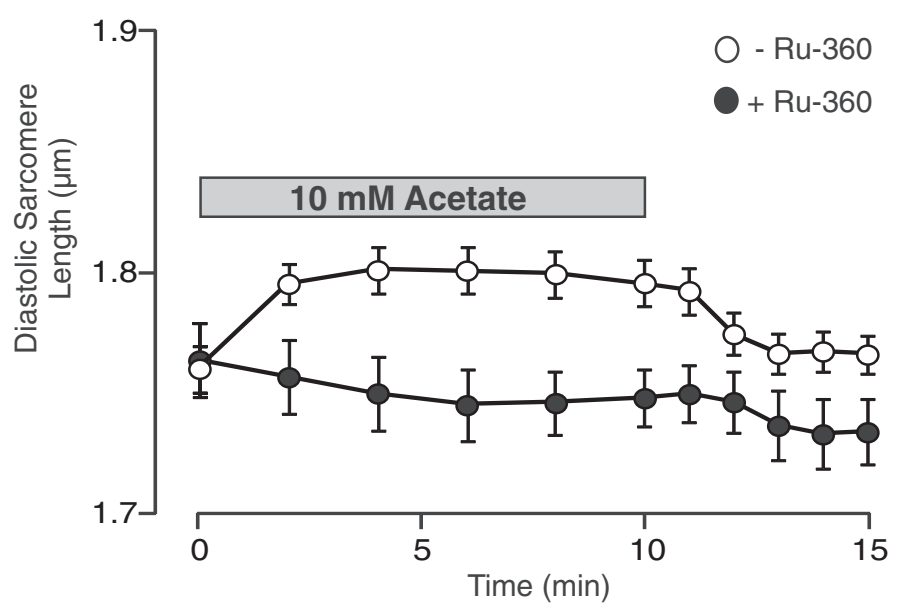

Figure 5 Inhibition of mitochondrial $\mathrm{Ca}^{2+}$ uptake with Ru-360 inhibits the effects of acetate on cell contraction. (A) Representative recording of average sarcomere length assessed continuously throughout application and removal of normal Tyrode solution (NT) supplemented with $10 \mathrm{mM}$ sodium acetate in cells pretreated with $10 \mu \mathrm{M}$ Ru-360 for 30-60 minutes and maintained in Ru-360 throughout the acetate exposure. Summary data from experiments as in $\mathbf{A}(n=11)$ illustrate that pretreatment with Ru-360 markedly suppresses the effect of acetate on both (B) fractional shortening and (C) diastolic sarcomere length.

not observed in experiments where myocytes were briefly exposed to thapsigargin prior to acetate. This finding suggests that the recovery of contraction during acetate treatment reflects refilling of the sarcomplasmic reticulum with $\mathrm{Ca}^{2+}$.

\section{Discussion}

Acetate and cardiac function

There have been previous studies that have examined the effects of acetate on cardiovascular function and energetics in different contexts. Consistent with the results 

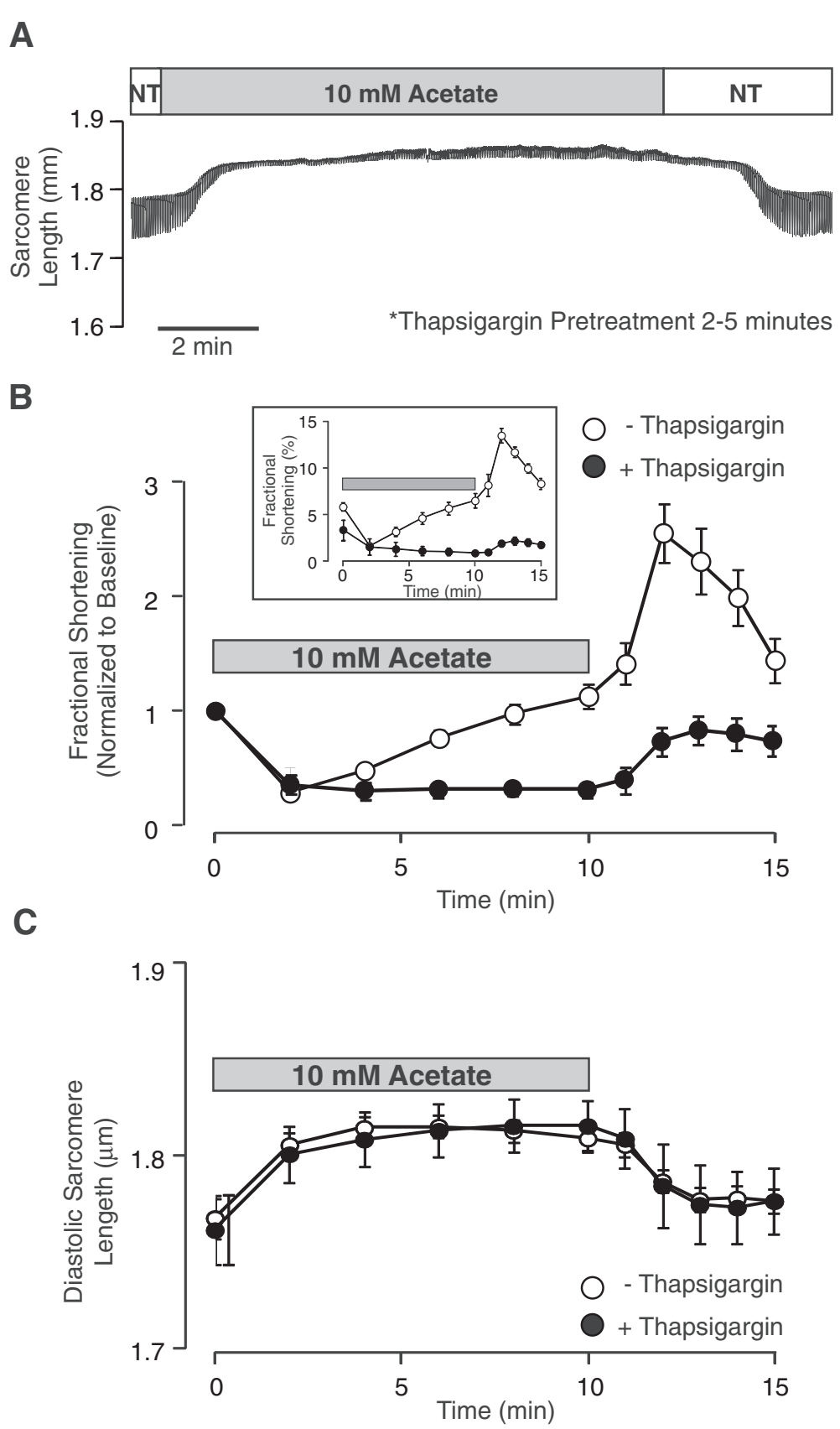

Figure 6 Thapsigargin pretreatment specifically impairs the recovery of contraction during acetate exposure. (A) Representative recording of average sarcomere length assessed continuously throughout application and removal of normal Tyrode solution (NT) supplemented with $10 \mathrm{mM}$ sodium acetate in cells pretreated with thapsigargin $(1 \mu \mathrm{M})$ for 2-5 minutes. As expected, cell relaxation was markedly slowed so contractions were evoked at $0.5 \mathrm{~Hz}$. (B \& C) Summary data from experiments as in $\mathbf{A}(n=9)$. Thapsigargin pretreatment had no effect on the acetate induced decrease in fractional shortening or increase in diastolic sarcomere length, but markedly blunted the recovery of contraction typically observed from 2-10 minutes during acetate application.

of the present study, acetate is reported to exhibit negative inotropic effects which appear to be transient $[4,5]$. It should also be noted that acetate infusion can also cause an acute reduction of the blood pressure that is most likely dependent on the increase in AMP associated with the conversion of acetate to acetyl $\mathrm{CoA}$ and local release of the vasodilator adenosine [6], complicating the interpretation of experiments in vivo. Nevertheless, the data in the present study indicate that acetate exerts effects on cardiac contraction by directly modulating myocyte function, at least partially independent of energy metabolism. 


\section{A possible role for mitochondrial $\mathrm{Ca}^{2+}$ uptake in regulating contractile function}

The present data provide evidence for a calcium-dependent mechanism linking acetate exposure with contractile function. Unlike the long chain fatty acid palmitate [2], the short chain fatty acid acetate caused no marked change in APD. Instead, we provide evidence that acetate caused a marked increased in mitochondrial $\mathrm{Ca}^{2+}$ accumulation. Moreover, the effect of acetate was markedly attenuated by pretreatment of myocytes with Ru-360, an inhibitor of mitochondrial $\mathrm{Ca}^{2+}$ uptake. Ru-360 is commonly used to inhibit mitochondrial $\mathrm{Ca}^{2+}$ uptake in a number of studies due to its ability to permeate cell membranes and it has been previously shown to have little if any effect on other transmembrane $\mathrm{Ca}^{2+}$ transport processes, including SR $\mathrm{Ca}^{2+}$ uptake and release, L-type $\mathrm{Ca}^{2+}$ channel and sodium calcium exchange function [19]. Our data is consistent with the conclusion that acetate uptake is coupled with an increase in mitochondrial $\mathrm{Ca}^{2+}$ and that this decreases, at least transiently, the amount of $\mathrm{Ca}^{2+}$ available for contraction of unloaded cardiomyocytes.

Interestingly, the effects of acetate on cell contraction were transient, with complete recovery of fractional shortening within 10 minutes of continuous exposure. This result could imply that as $\mathrm{Ca}^{2+}$ is sequestered in the mitochondria in the presence of acetate, additional $\mathrm{Ca}^{2+}$ enters the cell and refills the $\mathrm{SR} \mathrm{Ca}^{2+}$ stores. We found that the acute decrease in contraction amplitude and increase in diastolic sarcomere length were unaffected by pretreatment with thapsigargin to attenuate SERCA activity. Interestingly, however, the recovery of depressed myocyte contractility during sustained acetate exposure and the abrupt increase in contraction amplitude due to acetate removal were no longer observed when cells were pretreated with thapsigargin. One possible explanation of this phenomenon is that inhibition of $\mathrm{SR} \mathrm{Ca}^{2+}$ store replenishment prevents the restoration of fractional shortening.

\section{Acetate effects on mitochondria}

Mitochondrial uptake of acetate has been shown to be coupled with increases in $\mathrm{Ca}^{2+}$ uptake and swelling [13]. It was proposed that direct transport of phosphate or anions of weak acids like acetate can permeate the mitochondrial membrane generatings a driving force for $\mathrm{Ca}^{2+}$ uptake into the mitochondrial matrix. Similar observations demonstrate that acetate or phosphate increase the rate of mitochondrial $\mathrm{Ca}^{2+}$ uptake in a concentration dependent manner [14] and that acetate increases mitochondrial $\mathrm{Ca}^{2+}$ uptake in heart mitochondria [15]. The data in the present study do not delineate the molecular transporters mediating $\mathrm{Ca}^{2+}$ entry into the mitochondria. In addition to the mitochondrial $\mathrm{Ca}^{2+}$ uniporter [22], the mitochondrial ryanodine receptor (mRyr) [23], or $\mathrm{Ca}^{2+} / \mathrm{H}+$ exchanger (Letm1) [24] could play a role in the acetate-induced increase in mitochondrial $\mathrm{Ca}^{2+}$. Nevertheless, here we present pharmacological evidence showing that acetate is apparently coupled with an increase in mitochondrial $\mathrm{Ca}^{2+}$ in isolated cardiac myocytes and it is plausible to assume that this decreases the amount of $\mathrm{Ca}^{2+}$ available for contraction.

In isolated mitochondria, acetate also caused osmotic swelling [13]. Given the tight interfibrillar packing of mitochondria in the heart $[25,26]$, acetate associated mitochondrial swelling might also be linked to the observed increase in diastolic sarcomere length. Interestingly, the effect of acetate on diastolic sarcomere length, unlike the effect on contraction, is sustained throughout acetate exposure and shows little concentration dependence. This observation is consistent with the conclusion that acetate causes the mitochondria to swell increasing the sarcomere spacing. While active cell shortening recovers as the SR refills with $\mathrm{Ca}^{2+}$, the mitochondria remain swollen and the increased diastolic sarcomere length is maintained. Given the structural constraints, it seems possible that swelling is physically limited possibly explaining the absence of a clear concentration dependence.

The observed delay from acetate application to initial effects on contraction is similar to the time course for conversion of acetate to acetyl CoA observed by Randle [10]. Acetate has been shown to decrease the phosphorylation potential [27] resulting in decreased free energy from ATP hydrolysis; since the demand likely remains constant, the cell must increase the rate of respiration to cover the difference. This might suggest that acetate oxidation increases mitochondrial respiration with concomitant increases in mitochondrial $\mathrm{Ca}^{2+}$. However, this is very unlikely since substrate availability governs only the pathways used to generate ATP, while workload or demand is the principal determinant of respiration [12,28]. In unloaded myocytes paced at a constant frequency, it is expected that the workload is constant; therefore, it is unlikely that acetate-induced changes in respiration underlie the increase in mitochondrial $\mathrm{Ca}^{2+}$ uptake. Rather, we consider the possibility that mitochondrial uptake of the acetate anion is electrically balanced by the uptake of $\mathrm{Ca}^{2+}$ in line with the conclusion of others based on experiments with isolated mitochondria [13-15].

Short chain fatty acids like acetate and butyrate may also cause changes in intracellular $\mathrm{pH}$ with effects on contraction and $\mathrm{SR} \mathrm{Ca}^{2+}$ content $[29,30]$. The current data do not preclude a role for cytoplasm acidification in the effect of acetate on myocyte contraction. However, the observations that acetate increases mitochondrial $\mathrm{Ca}^{2+}$ and that pretreatment of cells with $\mathrm{Ru}-360$ markedly attenuates the effects of acetate argues that an acute change in mitochondrial $\mathrm{Ca}^{2+}$ uptake, rather than cytoplasmic acidification, is the predominant mechanism underlying the effects of acetate on contraction. Moreover, it is tempting to predict 
that other anions of weak organic acids (e.g. lactate, butyrate, or pyruvate) may cause similar changes.

\section{Mitochondrial $\mathrm{Ca}^{2+}$ uptake and cardiovascular disease}

In the heart, mitochondrial $\mathrm{Ca}^{2+}$ uptake has been proposed as an important player in regulating cardiac energetics, reactive oxygen species generation and supply-demand matching [31,32]; however, mitochondrial $\mathrm{Ca}^{2+}$ overload is also associated with the activation of cell death pathways [33]. Thus a balance in mitochondrial $\mathrm{Ca}^{2+}$ loading is required in order to achieve proper regulation of metabolism, but avoid overloading and cell death. In ischemia-reperfusion experiments, excessive $\mathrm{Ca}^{2+}$ uptake is associated with a poor outcome, and treatment with $\mathrm{Ru}-360$ to inhibit mitochondrial $\mathrm{Ca}^{2+}$ uptake is beneficial [34]. Acetate in this setting would be predicted to have no effect or be detrimental, and this has been shown to be the case [3,35-37]. Conversely, the failing heart has been shown to have reduced mitochondrial $\mathrm{Ca}^{2+}$ resulting from increases in cytosolic $\mathrm{Na}^{+}$concentrations and increased mitochondrial sodium-calcium exchange activity and blocking mitochondrial $\mathrm{Ca}^{2+}$ export (i.e. enhancing mitochondrial $\mathrm{Ca}^{2+}$ ) is beneficial [38]. The data in the present study suggest that elevating circulating acetate might be an alternative strategy to accomplish this goal, although it should be noted that the study presented here was focused on the transient and not steady state consequences of acetate.

\section{Conclusions}

In summary, we have shown that acetate causes an acute but transient reduction in contractile function in isolated cardiac myocytes. Mechanistically, the transient negative inotropic effect appears to result from an acetatedependent increase in mitochondrial $\mathrm{Ca}^{2+}$ uptake. This finding is consistent with the results of Lehninger and others using isolated liver and heart mitochondria, where it has been shown that acetate causes an increase in mitochondrial $\mathrm{Ca}^{2+}$ uptake and osmotic swelling [13-15]. Here we show that this effect appears to extend into intact, hydraulically unloaded cardiomyocytes, possibly suggesting a novel way to modulate mitochondrial $\mathrm{Ca}^{2+}$ homeostasis in the intact heart in vivo.

\section{Competing interests}

The authors declare that they have no competing interests.

\section{Authors' contributions}

JS performed the cell shortening experiments and analyzed data. AN participated in the design of the study and manuscript revision. $\mathrm{RC}$ performed mitochondrial $\mathrm{Ca}^{2+}$ uptake measurements and analyzed data. RB participated in the design of the study, data interpretation, and manuscript revision. TF performed electrophysiology experiments, cell shortening experiments, analyzed data, and drafted the manuscript. All authors read and approved the final manuscript.

\section{Acknowledgements}

This work was supported by funding from the Henry M. Jackson Foundation (to TPF) and the Department of Defense (R0702O to TPF).

\section{Disclaimer}

The views expressed are those of the authors and do not reflect the official policy or position of the Uniformed Services University of the Health Sciences, the Department of the Defense, or the United States governmen.

\section{Author details}

'Department of Anatomy, Physiology, and Genetics, Uniformed Services University for the Health Sciences, 4301 Jones Bridge Road, Rm. C-2114, Bethesda 20814, MD, USA. ${ }^{2}$ Department of Biochemistry and Molecular Biology, Uniformed Services University for the Health Sciences, Bethesda 20814, MD, USA

Received: 27 March 2014 Accepted: 24 November 2014

Published online: 09 December 2014

\section{References}

1. Flagg TP, Cazorla O, Remedi MS, Haim TE, Tones MA, Bahinski A, Numann RE, Kovacs A, Schaffer JE, Nichols CG, Nerbonne JM: Ca2 +-independent alterations in diastolic sarcomere length and relaxation kinetics in a mouse model of lipotoxic diabetic cardiomyopathy. Circ Res 2009, 104(1):95-103

2. Haim TE, Wang W, Flagg TP, Tones MA, Bahinski A, Numann RE, Nichols CG, Nerbonne JM: Palmitate attenuates myocardial contractility through augmentation of repolarizing Kv currents. J Mol Cell Cardiol 2010, 48(2):395-405.

3. Martin BJ, Valdivia HH, Bunger R, Lasley RD, Mentzer RM Jr: Pyruvate augments calcium transients and cell shortening in rat ventricular myocytes. Am J Physiol 1998, 274(1 Pt 2):H8-H17.

4. Kirkendol PL, Pearson JE, Bower JD, Holbert RD: Myocardial depressant effects of sodium acetate. Cardiovasc Res 1978, 12(2):127-136.

5. Jacob AD, Elkins N, Reiss OK, Chan L, Shapiro Jl: Effects of acetate on energy metabolism and function in the isolated perfused rat heart. Kidney Int 1997, 52(3):755-760.

6. Liang CS, Lowenstein JM: Metabolic control of the circulation. Effects of acetate and pyruvate. J Clin Invest 1978, 62(5):1029-1038.

7. Ballard FJ: Supply and utilization of acetate in mammals. Am J Clin Nutr 1972, 25(8):773-779.

8. Skutches CL, Holroyde CP, Myers RN, Paul P, Reichard GA: Plasma acetate turnover and oxidation. J Clin Invest 1979, 64(3):708-713.

9. Fujino T, Kondo J, Ishikawa M, Morikawa K, Yamamoto TT: Acetyl-CoA synthetase 2, a mitochondrial matrix enzyme involved in the oxidation of acetate. J Biol Chem 2001, 276(14):11420-11426.

10. Randle PJ, England PJ, Denton RM: Control of the tricarboxylate cycle and its interactions with glycolysis during acetate utilization in rat heart. Biochem J 1970, 117(4):677-695.

11. Williamson JR: Effects of insulin and starvation on the metabolism of acetate and pyruvate by the perfused rat heart. Biochem J 1964, 93(1):97-106.

12. Taegtmeyer $\mathrm{H}$, Hems $\mathrm{R}$, Krebs HA: Utilization of energy-providing substrates in the isolated working rat heart. Biochem J 1980, 186(3):701-711.

13. Lehninger AL: Role of phosphate and other proton-donating anions in respiration-coupled transport of $\mathrm{Ca} 2+$ by mitochondria. Proc Natl Acad Sci U S A 1974, 71(4):1520-1524.

14. Reed KC, Bygrave FL: A kinetic study of mitochondrial calcium transport. Eur J Biochem 1975, 55(3):497-504.

15. Harris EJ: Anion/calcium ion ratios and proton production in some mitochondrial calcium ion uptakes. Biochem J 1978, 176(3):983-991.

16. Flagg TP, Charpentier F, Manning-Fox J, Remedi MS, Enkvetchakul D, Lopatin A, Koster J, Nichols C: Remodeling of excitation-contraction coupling in transgenic mice expressing ATP-insensitive sarcolemmal K-ATP channels. Am J Physiol Heart Circ Physiol 2004, 286(4):H1361-H1369.

17. Pan X, Liu J, Nguyen T, Liu C, Sun J, Teng Y, Fergusson MM, Rovira Il, Allen M, Springer DA, Aponte AM, Gucek M, Balaban RS, Murphy E, Finkel T: The physiological role of mitochondrial calcium revealed by mice lacking the mitochondrial calcium uniporter. Nat Cell Biol 2013, 15(12):1464-1472. 
18. Miyata H, Silverman HS, Sollott SJ, Lakatta EG, Stern MD, Hansford RG: Measurement of mitochondrial free $\mathrm{Ca} 2+$ concentration in living single rat cardiac myocytes. Am J Physiol 1991, 261(4 Pt 2):H1123-H1134.

19. Matlib MA, Zhou Z, Knight S, Ahmed S, Choi KM, Krause-Bauer J, Phillips R, Altschuld R, Katsube Y, Sperelakis N, Bers DM: Oxygen-bridged dinuclear ruthenium amine complex specifically inhibits $\mathrm{Ca} 2+$ uptake into mitochondria in vitroand in situ in single cardiac myocytes. J Biol Chem 1998, 273(17):10223-10231.

20. Zhou Z, Bers D: Time course of action of antagonists of mitochondrial Ca uptake in intact ventricular myocytes. Pflugers Arch 2002, 445(1):132-138.

21. Bode EF, Briston SJ, Overend CL, O'Neill SC, Trafford AW, Eisner DA: Changes of SERCA activity have only modest effects on sarcoplasmic reticulum $\mathrm{Ca} 2+$ content in rat ventricular myocytes. J Physiol 2011, 589(19):4723-4729.

22. Marchi S, Pinton P: The mitochondrial calcium uniporter complex: molecular components, structure and physiopathological implications. J Physiol 2014, 592(Pt 5):829-839.

23. Beutner G, Sharma VK, Giovannucci DR, Yule DI, Sheu SS: Identification of a ryanodine receptor in rat heart mitochondria. J Biol Chem 2001, 276(24):21482-21488.

24. Jiang D, Zhao L, Clapham DE: Genome-Wide RNAi Screen Identifies Letm1 as a Mitochondrial Ca2+/H+ Antiporter. Science 2009, 326(5949):144-147.

25. Lukyanenko V, Chikando A, Lederer WJ: Mitochondria in cardiomyocyte Ca2+ signaling. Int J Biochem Cell Biol 2009, 41(10):1957-1971.

26. Ong S-B, Hausenloy DJ: Mitochondrial morphology and cardiovascular disease. Cardiovasc Res 2010, 88(1):16-29.

27. Kang YH, Mallet RT, Bunger R: Coronary autoregulation and purine release in normoxic heart at various cytoplasmic phosphorylation potentials: disparate effects of adenosine. Pflugers Arch 1992, 421(2-3):188-199.

28. Neely JR, Denton RM, England PJ, Randle PJ: The effects of increased heart work on the tricarboxylate cycle and its interactions with glycolysis in the perfused rat heart. Biochem J 1972, 128(1):147-159.

29. Bountra C, Vaughan-Jones RD: Effect of intracellular and extracellular pH on contraction in isolated, mammalian cardiac muscle. J Physiol 1989, 418:163-187.

30. O'Neill SC, Eisner DA: pH-dependent and -independent effects inhibit $\mathrm{Ca} 2+-$-induced $\mathrm{Ca} 2$ + release during metabolic blockade in rat ventricular myocytes. J Physiol 2003, 550(2):413-418.

31. Balaban RS: Cardiac energy metabolism homeostasis: role of cytosolic calcium. J Mol Cell Cardiol 2002, 34(10):1259-1271.

32. Liu T, O'Rourke B: Regulation of mitochondrial Ca2+ and its effects on energetics and redox balance in normal and failing heart. I Bioenerg Biomembr 2009, 41(2):127-132.

33. Garcia-Dorado D, Ruiz-Meana M, Inserte J, Rodriquez-Sinovas A, Piper HM: Calcium-mediated cell death during myocardial reperfusion. Cardiovasc Res 2012, 94(2):168-180.

34. Garcia-Rivas Gde J, Carvajal K, Correa F, Zazueta C: Ru360, a specific mitochondrial calcium uptake inhibitor, improves cardiac post-ischaemic functional recovery in rats in vivo. Br J Pharmacol 2006, 149(7):829-837.

35. Mallet RT, Sun J, Knott EM, Sharma AB, Olivencia-Yurvati AH: Metabolic cardioprotection by pyruvate: recent progress. Exp Biol Med (Maywood) 2005, 230(7):435-443.

36. Bunger R, Mallet RT: Mitochondrial pyruvate transport in working guinea-pig heart. Work-related vs carrier-mediated control of pyruvate oxidation. Biochim Biophys Acta 1993, 1151(2):223-236

37. Bunger R, Mallet RT, Hartman DA: Pyruvate-enhanced phosphorylation potential and inotropism in normoxic and postischemic isolated working heart. Near-complete prevention of reperfusion contractile failure. Eur J Biochem 1989, 180(1):221-233.

38. Liu T, O'Rourke B: Enhancing mitochondrial Ca2+ uptake in myocytes from failing hearts restores energy supply and demand matching. Circ Res 2008, 103(3):279-288.

doi:10.1186/s12899-014-0012-2

Cite this article as: Schooley et al: Acetate transiently inhibits myocardial contraction by increasing mitochondrial calcium uptake. BMC Physiology 2014 14:12

\section{Submit your next manuscript to BioMed Central and take full advantage of:}

- Convenient online submission

- Thorough peer review

- No space constraints or color figure charges

- Immediate publication on acceptance

- Inclusion in PubMed, CAS, Scopus and Google Scholar

- Research which is freely available for redistribution

Submit your manuscript at www.biomedcentral.com/submit
() Biomed Central 